\title{
17 $\alpha$-hydroxylase/17,20-lyase deficiency in congenital adrenal hyperplasia: A case report
}

\author{
SIMIAO XU, SHUHONG HU, XUEFENG YU, MUXUN ZHANG and YAN YANG \\ Department of Endocrinology, Tongji Hospital, Tongji Medical College, \\ Huazhong University of Science and Technology, Wuhan, Hubei 430030, P.R. China
}

Received November 14, 2015; Accepted November 11, 2016

DOI: $10.3892 / \mathrm{mmr} .2016 .6029$

\begin{abstract}
Congenital adrenal hyperplasia (CAH) is a rare autosomal recessive disorder caused by mutations in the cytochrome P450 family 17 subfamily A member 1 (CYP17A1) gene located on chromosome 10q24.3, which leads to a deficiency in $17 \alpha$-hydroxylase/17,20-lyase. The disorder is characterized by low blood levels of estrogens, androgens and cortisol, which leads to a compensatory increase in adrenocorticotropic hormone levels that stimulate the production of mineralocorticoid precursors. This subsequently leads to hypertension, hypokalemia, primary amenorrhea and sexual infantilism. Over 90 distinct genetic lesions have been identified in patients with this disorder. The prevalence of common mutation of CYP17Al gene differs among ethnic groups. Treatment of this disorder involves replacement of glucocorticoids and sex steroids. Estrogen alone is prescribed for patients who are biologically male with $17 \alpha$-hydroxylase deficiencies that identify as female. However, genetically female patients may receive estrogen and progesterone supplementation. In the present study, a 17-year-old female with 17 $\alpha$-hydroxylase/17,20-lyase deficiency that presented with primary amenorrhea and sexual infantilism and no hypertension, was examined. The karyotype of the patient was $46, \mathrm{XX}$, and genetic analysis revealed the presence of a compound heterozygous mutation in exons 6 and 8 , leading to the complete absence of $17 \alpha$-hydroxylase/17,20-lyase activity. The patient was treated with prednisolone and ethinyl estradiol. In addition, a summary of the recent literature regarding $\mathrm{CAH}$ is presented.
\end{abstract}

Correspondence to: Dr Yan Yang, Department of Endocrinology, Tongji Hospital, Tongji Medical College, Huazhong University of Science and Technology, 1095 Jiefang Avenue, Wuhan, Hubei 430030, P.R. China

E-mail: yangyantjh@gmail.com

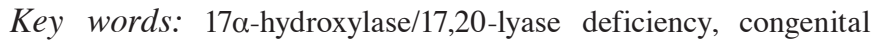
adrenal hyperplasia, gene mutation, primary amenorrhea

\section{Introduction}

The endocrine disease, congenital adrenal hyperplasia $(\mathrm{CAH})$, defines a group of syndromes resulting from inherited defects in one of the five enzymes involved in the biosynthesis of cortisol from cholesterol. These enzymes include $21 \alpha$-hydroxylase, $11 \beta$-hydroxylase and 17 $\alpha$-hydroxylase/17,20-lyase, which are associated with P450C21, P45011B, and P45017A1 forms of cytochrome P450, as well as cholesterol 20,22 desmolase and $3 \beta$-hydroxysteroid dehydrogenase (1). As a result of this enzymatic defect, cortisol levels decrease and the negative feedback control of adrenocorticotropic hormone (ACTH) levels is absent, which leads to excess ACTH production to normalize cortisol levels (2). This results in overproduction and accumulation of steroid precursors and high ACTH levels that lead to adrenal hyperplasia. The clinical manifestation of the disorder depends on the levels of these intermediates in the steroid biosynthetic pathway for the blockade of any one of the steps (2). CAH should be considered in infants, children or adolescents in the clinic when evaluating ambiguous genitalia, sexual infantilism, hypogonadism or hypertension, particularly when associated with disturbed fluid, electrolyte and hydrogen homeostasis (3). The most common form of CAH involves 21-hydroxylase deficiency, which accounts for $90-95 \%$ of $\mathrm{CAH}$ cases, whilst $17 \alpha$-hydroxylase/17,20-lyase deficiency is the least common form of the disorder, $<1 \%$ of all CAH cases (4).

$17 \alpha$-hydroxylase/17,20-lyase deficiency is caused by mutations in the cytochrome P450 family 17 subfamily A member 1 (CYP17A1) gene, and is associated with the impaired production of cortisol and sex steroids (5). The deficiency leads to elevation of plasma ACTH levels and accumulation of mineralocorticoids, thus resulting in hypertension, hypokalemia and bilateral adrenal hyperplasia (5). In addition, the impaired production of sex steroids leads to ambiguous or female external genitalia in affected male individuals (46, XY), and normal genitalia in affected females $(46, \mathrm{XX})$ at birth, but no sexual development at the expected time of puberty $(6,7)$.

The current study presents a case of a female Chinese patient (age, 17 years) with a deficiency in $17 \alpha$-hydroxylase/17,20-lyase caused by a compound heterozygous mutation (c.985-987 in exon 6, c.1459-1467 in exon 8) in the CYP17A1 gene. 
Table I. Plasma steroid and pituitary hormone levels in the patient, and 17 $\alpha$-hydroxyprogesterone levels in the patient's family members at clinical presentation.

\begin{tabular}{|c|c|c|}
\hline Plasma steroid/pituitary hormone & Result & Reference range \\
\hline FSH $(\mathrm{mIU} / \mathrm{ml})$ & 104.41 & Follicular, 3.85-8.78; ovulatory, 4.54-22.51; luteal, 1.79-5.12 \\
\hline $\mathrm{LH}(\mathrm{mIU} / \mathrm{ml})$ & 26.09 & $\begin{array}{l}\text { Follicular, 2.12-10.89; ovulatory, 19.18-103.03; luteal, } \\
1.20-12.86\end{array}$ \\
\hline Estradiol (pg/ml) & $<20$ & Follicular, 24-114; ovulatory, 62-534; luteal, 80-273 \\
\hline Progesterone (ng/ml) & 9.89 & Follicular, 0.31-1.52; luteal, 5.16-18.56 \\
\hline Testosterone (ng/ml) & $<0.1$ & $0.10-0.75$ \\
\hline Prolactin (ng/ml) & 12.32 & $3.34-26.72$ \\
\hline SHBG (nmol/l) & 104 & $18.2-135.5$ \\
\hline $\mathrm{GH}(\mathrm{ng} / \mathrm{ml})$ & 0.354 & $0-10$ \\
\hline IGF-1 (ng/ml) & 236 & $412 \pm 80$ \\
\hline DHEA-S (nmol/1) & $<1$ & $3540 \pm 1310$ \\
\hline Androstenedione (nmol/l) & $<0.01$ & Follicular, $2.7 \pm 1$; luteal, $5.2 \pm 1.5$ \\
\hline $17 \alpha$-hydroxyprogesterone (nmol/l) & 1.07 & Follicular, $1.3 \pm 0.25$; luteal, $7.4 \pm 2$ \\
\hline $\mathrm{ACTH}(\mathrm{pmol} / \mathrm{l})$ & 15.94 & $1.6-13.9$ \\
\hline Cortisol $(\mu \mathrm{g} / \mathrm{l})$ 8:00 a.m. & 35.44 & $62-194$ \\
\hline Cortisol $(\mu \mathrm{g} / \mathrm{l})$ 4:00 p.m. & 34.83 & $23-123$ \\
\hline Renin concentration $(\mathrm{ng} / \mathrm{ml} / \mathrm{h})$ & $<0$ & Recumbent, $0.05-0.79$; upright, $0.93-6.56$ \\
\hline Aldosterone (ng/dl) & 16.5 & Recumbent, 5.9-17.4; upright, 6.5-29.6 \\
\hline Father: $17 \alpha$-hydroxyprogesterone (nmol/1) & 8.69 & $3.5 \pm 1.2$ \\
\hline Mother: $17 \alpha$-hydroxyprogesterone (nmol/l) mother & 1.88 & Follicular, $1.3 \pm 0.25$; luteal, $7.4 \pm 2$ \\
\hline Sister: $17 \alpha$-hydroxyprogesterone (nmol/l) & 2.21 & Follicular, $1.3 \pm 0.25$; luteal, $7.4 \pm 2$ \\
\hline
\end{tabular}

FSH, follicle-stimulating hormone; LH, luteinizing hormone; SHBG, sex hormone-binding globulin; GH, growth hormone; IGF-1, insulin-like growth factor 1; DHEA-S, dehydroepiandrosterone sulfate; ACTH, adrenocorticotropic hormone.

\section{Case report}

A Chinese woman (age, 17 years) was transferred from Shishou Hospital to the Department of Endocrinology at Tongji Hospital (Wuhan, China) in June 2015, and presented with primary amenorrhea and evidence of sexual infantilism. The patient's parents noticed a lack of breast development, as well as an absence of axillary and pubic hair since the beginning of puberty. The patient's parents were non-consanguineous and reported no history of hypertension or recurrent episodes of periodic paralysis. There was no reported history of surgery or additional diseases in the patient. In addition, the patient's 10 -year-old sister appeared normal as she presented normal female sex characteristics, no hypertension and no periodic paralysis.

Upon physical examination, the patient was tall and slender, with a height of $163 \mathrm{~cm}$, a weight of $45 \mathrm{~kg}$ and a body mass index of $16.9 \mathrm{~kg} / \mathrm{m}^{2}$. Blood pressure was normal $(128 / 99 \mathrm{mmHg})$, and the patient's skin color was slightly darker when compared with that of her parents. The patient's breast development had only progressed to Tanner stage B1P1 and external genitalia were phenotypically female, but were infantile. A wide palpebral fissure and a slightly thick upper lip was evident, however there was no webbed neck or cubitus valgus. Cardiovascular system examination revealed normal heart sounds and muscular tension test exhibited grade 5 strength.
Routine laboratory tests revealed a normal white blood cell count, hemoglobin, platelet count, coagulation function, urine (specific gravity, $\mathrm{PH}$, protein, glucose and microscopic analysis) and stools. Blood biochemistry analysis revealed results within the reference ranges for liver function, renal function, glucose, lipids and thyroid profiles, with low serum potassium $(3.09 \mathrm{mmol} / 1$, normal range, $3.50-5.10 \mathrm{mmol} / \mathrm{l})$ and mild alkalosis $(\mathrm{pH} 7.39$, normal range, 7.350-7.450; bicarbonate $26 \mathrm{mmol} / \mathrm{l}$, normal range, 21.3-24.8 mmol/l). Pelvic ultrasonography revealed a primordial uterus $(1.9 \times 0.5 \mathrm{~cm})$ and hypoplastic ovaries (left, $1.2 \times 0.6 \mathrm{~cm}$, right, $1.6 \times 0.7 \mathrm{~cm}$ ). Magnetic resonance imaging demonstrated that the pituitary gland was normal, and bilateral integration of the adrenal glands was enlarged, as determined by enhanced computed tomography. Ambulatory blood pressure monitoring demonstrated that blood pressure was in the normal range (average, $127 / 85 \mathrm{mmHg}$ ). However, the patient's bone age was delayed (age, 12 years). Chromosomal analysis revealed a normal 46, XX female karyotype.

The patient received endocrine tests, and the results of blood analysis for adrenal, gonadal and pituitary hormones, as well as the renin-angiotensin-aldosterone system are presented in Table I. The patient presented with low serum cortisol levels and high levels of ACTH, which were consistent with the results upon re-examination after 1 week. Follicle-stimulating hormone (FSH) and luteinizing hormone (LH) levels were elevated, but estradiol and testosterone 
were low, suggesting a diagnosis of primary gonadal failure (hypergonadotrophic hypogonadism). In addition, the patient presented with low $17 \alpha$-hydroxyprogesterone levels (the precursor of cortisol), and low levels of adrenal androgens [dehydroepiandrosterone-sulfate (DHEA-S)] and androstenedione (Table I). The renin-angiotensin-aldosterone system test demonstrated that the patient exhibited normal aldosterone levels and undetectable plasma renin activity. Blood profiles of the patient's parents and sister demonstrated that they all exhibited normal $17 \alpha$-hydroxyprogesterone levels, and the sister had a normal 46, XX karyotype.

The clinical manifestations, imaging and laboratory results appeared to be consistent with a diagnosis of $\mathrm{CAH}$ in the patient, due to the observed $17 \alpha$-hydroxylase deficiency. In order to confirm this diagnosis, genetic analysis of the CYP17Al sequence was performed on the patient and her family members.

Genomic DNAs were extracted from the peripheral blood leukocytes using the QIAamp DNA Blood Mini kit (Qiagen, Hilden, Germany) per the manufacturer's protocol. DNA was diluted to a final concentration of $10 \mathrm{ng} / \mu \mathrm{l}$ for use. All eight exons of CYP17A1 gene were amplified by polymerase chain reaction (PCR) using eight pairs of primers (Table II), which were designed with Primer Premier 5.0 software. The PCR was performed in a volume of $50 \mu \mathrm{l}$. Amplifications were sequenced with the BigDye Terminator v3.1 Cycle Sequence kit (Applied Biosystems; Thermo Fisher Scientific, Inc., Waltham MA, USA) using an ABI3130xl Genetic Analyzer (Applied Biosystems; Thermo Fisher Scientific, Inc.). Finally, the Chromas Lite 2.01 software was used to identify mutation candidates, which was confirmed by two independent observers.

Direct sequencing of PCR products revealed that the patient harbored the following two compound heterozygous mutations in the CYP17A1 gene: c.985-987 (TAC>AA) in exon 6, leading to amino acid alterations Y329K418X, a premature termination codon; and c.1459-1467 (GACTCTTTC deletion) in exon 8, leading to deletion of D487-S488-F489 amino acids. Analysis of DNA from the patient's parents and sister, demonstrated that the TAC $>$ AA mutation was present in the father, while the $9 \mathrm{bp}$ deletion was present in the mother. Fortunately, the patient's sister was homozygous wild-type alleles from her parents (Fig. 1).

Oral prednisolone treatment was started at $5 \mathrm{mg} / \mathrm{day}$, and estrogen replacement was provided to induce female secondary sexual development with oral estradiol (Progynova, $1 \mathrm{mg}$ daily). One year after the diagnosis, the patient's breast development progressed to Tanner stage B2P2, and she was still amenorrhea and had infantile external genitalia. The treatment was maintained.

The written informed consent was obtained from the patient and her family for the use of their samples in the current study.

\section{Discussion}

$17 \alpha$-hydroxylase deficiency is a rare form of CAH, with an estimated incidence of 1 in 50,000-100,000 individuals and represents $\sim 1 \%$ of all $\mathrm{CAH}$ cases $(8,9)$. $\mathrm{CAH}$ is an autosomal recessive disorder that results in decreased production of cortisol, androgens and estrogens, with a subsequent increase
Table II. Primers for CYP17A1 amplification.

\begin{tabular}{|c|c|c|}
\hline Exon & Sequence & $\begin{array}{l}\text { Product } \\
\text { size (bp) }\end{array}$ \\
\hline \multirow[t]{2}{*}{1} & F 5' CTTGTGCCCTAGAGTGCCA 3' & \\
\hline & R 5' GAAGGGGGCAGGGAGGAG 3' & 401 \\
\hline \multirow[t]{2}{*}{2} & F 5' GAAGGAAAGCAGGGACCAGA 3' & \\
\hline & R 5' GGCAGCAGTAGCCAAGAAAA 3' & 350 \\
\hline \multirow[t]{2}{*}{3} & F 5' CATCTGCTATCTGTCCCCCG 3' & \\
\hline & R 5' GGCTGGAGCAGGGAAGTAAA 3' & 419 \\
\hline \multirow[t]{2}{*}{4} & F 5' GCCCTTTGTCCTTTCCCTCA 3' & \\
\hline & R 5' GGGAACGAAAGGGGTGCTAA 3' & 468 \\
\hline \multirow[t]{2}{*}{5} & F 5'AGTCAGGGACAGAAGTATGGCAG 3' & \\
\hline & R 5' TGCACAGAAAGCCTGAGAGAATT 3' & 389 \\
\hline \multirow[t]{2}{*}{6} & F 5' GGAAGGGACTGGACAGGCTC 3' & \\
\hline & R 5' TGAATGCATCATGGGGCTAGA 3' & 324 \\
\hline \multirow[t]{2}{*}{7} & F 5' AAGGGCATTTTCCTCACGG 3' & \\
\hline & F 5' TTGGCAGAGGTGAAGGGGTA 3' & 291 \\
\hline \multirow[t]{2}{*}{8} & F 5' CTCAACCAGGGCAGAACCAT 3' & \\
\hline & R 5' GGTGGGGGGTTGTATCTCTAAA 3' & 429 \\
\hline
\end{tabular}

F, forward; R, reverse.

in ACTH and gonadotropin levels. The majority of patients with CAH typically present with hypertension and primary gonadal failure during adolescence and adulthood. However, a few individuals are reported to be normotensive at the time of diagnosis $(10,11)$. 17 $\alpha$-hydroxylase deficiency was first described in a 35-year-old patient in 1966 by Biglieri et al (12), who was phenotypically female and presented with hypertension, sexual infantilism and primary amenorrhea. There have been $>200$ cases reported in the literature.

The human CYP17Al gene is located on chromosome 10q24.3, and encodes the P450c17 enzyme. The gene spans $6.6 \mathrm{~kb}$, consists of eight exons and has a coding region of $1.6 \mathrm{~kb}(13,14)$. The P450c17 enzyme catalyzes steroid $17 \alpha$-hydroxylase and 17,20-lyase activities in the adrenal glands and gonads. Individuals with $\mathrm{CAH}$ that present with a deficiency in $17 \alpha$-hydroxylase should hypothetically be divided into the following three groups: i) Loss of $17 \alpha$-hydroxylase activity; ii) loss of 17,20-lyase; iii) the combined loss of $17 \alpha$-hydroxylase/17,20-lyase. However, it is often difficult to distinguish these forms from clinical and biochemical results, and a combined deficiency in both enzymes account for the majority of patients with CAH. In addition, 17,20-lyase deficiency is described in literature less frequently than $17 \alpha$-hydroxylase deficiency $(11,15)$.

In the steroid biosynthesis pathway, $17 \alpha$-hydroxylase/17, 20-lyase serves an essential role in cortisol and sex steroid production (Fig. 2). A deficiency in $17 \alpha$-hydroxylase/17,20 lyase, caused by a mutation in the CYP17Al gene, results in decreased synthesis of glucocorticoids and androgens (DHEA and androstenedione). In the biosynthetic pathway, cholesterol is first converted to pregnenolone. Pregnenolone is then converted to $17 \alpha$-hydroxypregnenolone by $17 \alpha$-hydroxylase, leading to its conversion, via the $3 \beta$-hydroxysteroid dehydrogenase pathway, to progesterone. In patients with $\mathrm{CAH}$ 

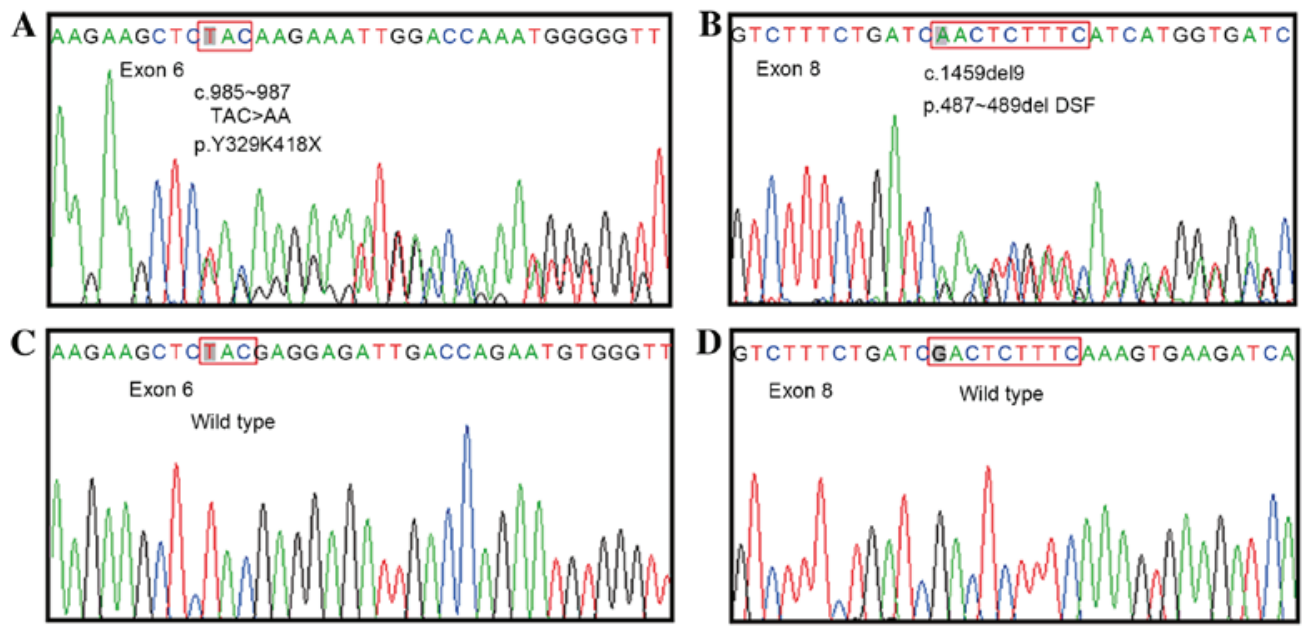

$\mathbf{E}$

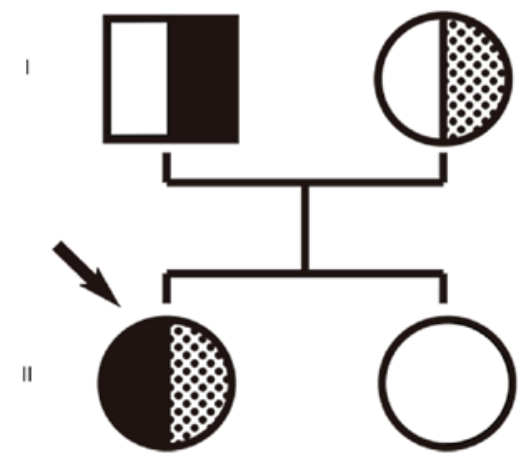

Figure 1. Pedigree analysis of the $C Y P 17 A 1$ gene. The patient was demonstrated to harbor compound heterozygous mutations in $C Y P 17 A 1$, whereas the parents each harbored a single heterozygous mutation and the sister harbored two wild-type alleles. (A) A heterozygous mutation (TAC $>$ AA) at position $985-987$ in exon 6 was detected in the patient and father. (B) A heterozygous mutation (GACTCTTTC deletion) at position 1459-1467 in exon 8 was detected in the patient and mother. (C) Wild-type copies of exon 6 were detected in the patient's mother and sister. (D) Wild-type copies of exon 8 were detected in the patient's father and sister. (E) A schematic representation of the pedigree of the family. The patient (as indicated by the black arrow) harbored a compound mutation, derived from the paternal and maternal lines. CYP17A1, cytochrome P450 family 17 subfamily A member 1.

\section{Adrenal steroidogenesis pathway}

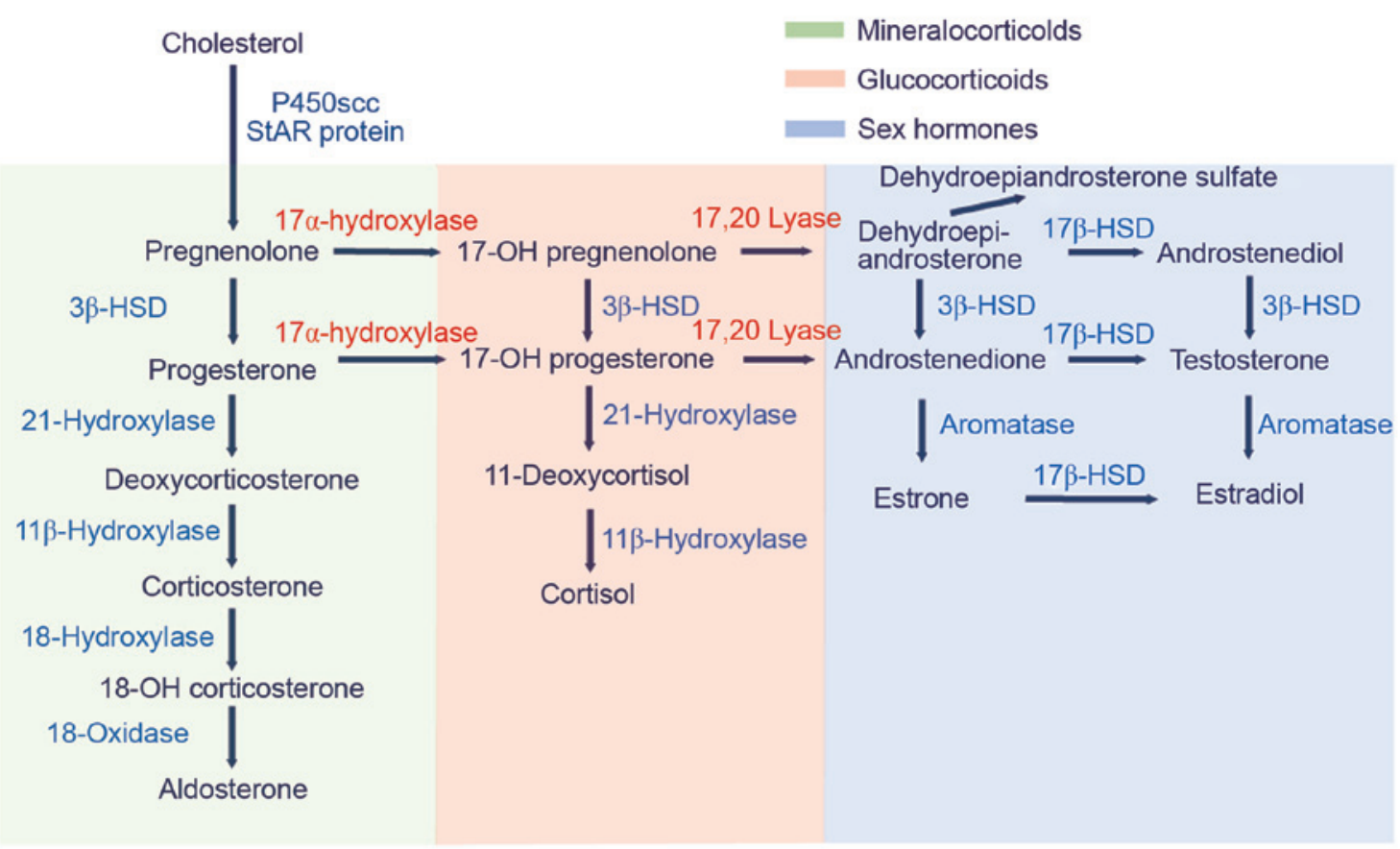

Figure 2. Schematic of the adrenal steroidogenesis biosynthesis pathway. HSD, hydroxysteroid dehydrogenase. 
that harbor 17,20-lyase mutations, progesterone cannot be $17 \alpha$-hydroxylated to $17 \alpha$-hydroxyprogesterone, as further conversion of these $17 \alpha$-hydroxylated derivatives to DHEA requires 17,20-lyase. In these patients, a decrease in $17 \alpha$-hydroxypregnenolone, $17 \alpha$-hydroxylprogesterone and DHEA occurs, which leads to a subsequent reduction in the production of steroids, including androstenedione, testosterone, 11-deoxycortisol and cortisol. Accumulation of the substrates, pregnenolone and progesterone, enhances the 21 - and $11 \beta$-hydroxylation steps in the mineralocorticoid pathway, resulting in an increase in 11-deoxycorticosterone, corticosterone and 18-hydroxycorticosterone levels $(1,2,16)$. In healthy individuals, decreased cortisol synthesis and the subsequent feedback inhibition on pituitary ACTH leads to an increase in ACTH release, in order to return cortisol production to normal levels. However, in $\mathrm{CAH}$ patients there is an overstimulation of the steroid synthetic pathway, which leads to the overproduction of mineralocorticoid precursors and hyperplasia of the adrenal cortex. High levels of mineralocorticoid precursors (11-deoxycorticosterone, corticosterone and 18-hydroxycorticosterone) induce sodium and fluid retention, and loss of potassium and hydrogen, which consequently induces hypertension and hypokalemic alkalosis, due to its potent mineralocorticoid effect (17). This feedback suppresses the renin-angiotensin-aldosterone system, causing hyporeninemic hypoaldosteronism or hyporeninemic normoaldosteronism (17). 17 $\alpha$-hydroxylase/17,20 lyase and $3 \beta$-hydroxysteroid dehydrogenase are present in the adrenal glands and gonads and contribute to sexual maturity throughout fetal life and puberty (18). Therefore, there is impairment of adrenal and gonadal steroid hormone production (androgens and estrogens) in patients with $17 \alpha$-hydroxylase/17,20 lyase deficiency. Affected male individuals have ambiguous or female external genitalia, however, they present with no internal male genitalia (prostate, seminal vesicles and vas deferens), which leads to an absence of testosterone (19). Female patients present with normal genitalia, but undergo immature sexual development and develop primary or secondary amenorrhea due to estrogen deficiency (19). However, the observed decline in cortisol levels does not manifest as classical Addison's disease, as raised corticosterone production demonstrates a mild glucocorticoid effect (10).

Based on the clinical, biochemical and molecular features, patients may be diagnosed with $\mathrm{CAH}$ due to a $17 \alpha$-hydroxylase/17,20 lyase deficiency. In the current case, biochemical detection demonstrated that there were decreased concentrations of estradiol, testosterone, DHEA, androstenedione and cortisol, as well as increased concentrations of progesterone and ACTH. The low levels of estradiol and testosterone led to infantile female genitalia, a lack of secondary sexual characteristics and primary amenorrhea, as well as an above-average height. The high levels of FSH and $\mathrm{LH}$, decreased cortisol and increased ACTH, indicated that primary gonadal failure and primary adrenal hypocortisolism were evident. Mild hypokalemic alkalosis may have occurred as a result of mineralocorticoid pathway activation, which led to renin suppression. However, the patient's aldosterone levels were within the normal range (10). Notably, the patient exhibited normotension, which accounts for $10-15 \%$ of patients that harbor mutations in the CYP17A gene in previous reports $(10,11)$.

To the best of our knowledge, 100 different CAH-associated mutations that lead to $17 \alpha$-hydroxylase/17,20-lyase deficiency have been identified (16). These mutations include missense and nonsense mutations, insertions, deletions and splice-site variants. The most common mutation sites differ among ethnic groups, which can be explained by the founder effect in autosomal recessive genetic disease (20). The patient examined in the present study was confirmed to harbor a compound heterozygous mutation (Y329K418X and D487-S488-F489 deletion) in the CYP17A1 gene by mutation analysis, which led to the complete loss of $17 \alpha$-hydroxylase/17,20-lyase activity. These mutations have been reported previously in Chinese $17 \alpha$-hydroxylase/17,20-lyase-deficient patients (21). The c.985-987 mutation (TAC>AA) has been detected in subjects from Korea and China (initially in a 32-year-old Korean female in 2003) (22), and the c.1459-1467 mutation (GACTCTTTC deletion) has been detected in subjects from Thailand and China (initially in a 14-year-old female from Thailand in 1993) (23). It has been reported that these mutations in exons 6 and 8 are prevalent in Chinese patients with 17 $\alpha$-hydroxylase/17,20-lyase deficiency, and may be major causes of $17 \alpha$-hydroxylase deficiency in the Chinese population (21)

Treatments for $17 \alpha$-hydroxylase/17,20-lyase deficiency include appropriate glucocorticoid replacement and sex steroid hormone supplementation. The aim of glucocorticoid treatment is to reduce ACTH and 11-deoxycorticosterone to normal levels, using a physiological dose of $0.25-1.0 \mathrm{mg}$ /day dexamethasone and 2-5 mg/day prednisone. The administration of glucocorticoids normalizes blood pressure, serum renin and electrolyte levels with natriuresis. In addition, sex hormone replacement therapy is recommended to be administered in adolescence for secondary sexual development, maintenance of female sexual characteristics and stimulation of epiphyseal closure. Estrogen and progestin cyclic therapy is required in $46, \mathrm{XX}$ patients to induce cyclic withdrawal bleeding and prevent endometrial hyperplasia. In addition, estrogen alone may often be prescribed when patients are admitted at the time of puberty, at the time of diagnosis in patients that are postpubescent, or when a male $(46, \mathrm{XY})$ wishes to be considered female. If a patient decides to be considered male, androgen replacement therapy is prescribed and extensive genital reconstructive surgery, such as a gonadectomy to avoid malignant degeneration in the intra-abdominal testes, may be performed. This treatment should be discussed with the patient and parents, with an emphasis on its complexity, implications and consequences $(5,24)$. In the present case, the patient was administered with prednisone $(5 \mathrm{mg} /$ day $)$ and estradiol valerate $(1 \mathrm{mg} /$ day). Despite this sex steroid replacement, patients with $17 \alpha$-hydroxylase/17,20-lyase deficiency develop hypergonadotropic hypogonadism and infertility due to the decreased enzymatic activity of $C Y P 17 A 1$. Irreversible defects in steroidogenesis may lead to impaired spermatogenesis and folliculogenesis, and therefore women are unable to conceive spontaneously or via endocrinological intervention (25). However, a single report of pregnancy in a patient with partial $17 \alpha$-hydroxylase/17,20-lyase deficiency, resulted in the live birth of triplets following in vitro fertilization cycles 
and transfer of cryopreserved embryos (26). However, to the best of our knowledge, further attempts at fertility therapy in individuals with $17 \alpha$-hydroxylase/17,20-lyase deficiency have not yet resulted in live births (25).

In conclusion, $17 \alpha$-hydroxylase/17,20-lyase deficiency is a rare, unusual and challenging to diagnose endocrine disorder. This case manifested non-typical clinical symptoms of $17 \alpha$-hydroxylase/17,20-lyase deficiency (e.g. no hypertension). To the best of our knowledge the present study was the first to demonstrate that the two compound heterozygous mutations in the CYP17A1 gene may be used for the diagnosis in the $17 \alpha$-hydroxylase/17,20-lyase deficient patient without typical clinical symptoms. In the majority of patients, the presentation of clinical symptoms may be delayed until puberty, and this subtype of CAH may not be diagnosed at birth. Therefore, some degree of clinical suspicion is necessary when evaluating children and adolescents with hypertension and hypokalemia, particularly when combined with the presence of sexual infantilism, and genetic analysis is recommended. In the current study, a biochemically and genetically proven case of 17a-hydroxylase/17,20 lyase deficiency with 46, XX karyotype was presented. The patient presented with mild hypokalemia, primary amenorrhea and sexual infantilism, but no hypertension. Diagnosis of $17 \alpha$-hydroxylase/17,20 lyase deficiency was confirmed by the characteristic profile of adrenal steroid levels, with further confirmation by CYP17A mutation analysis. Additional analysis of clinical, laboratory and molecular features may increase our understanding of the $\mathrm{CAH}$ endocrine disease.

\section{Acknowledgments}

The authors would like to thank Dr Chen Chen (Institute of Hypertension and Department of Internal Medicine, Tongji Hospital, Tongji Medical College, Huazhong University of Science and Technology, Wuahn, China) for her contribution to perform genetic analysis of the patient and her family members.

\section{References}

1. Honour JW: Diagnosis of diseases of steroid hormone production, metabolism and action. J Clin Res Pediatr Endocrinol 1: 209-226, 2009.

2. Miller WL and Auchus RJ: The molecular biology, biochemistry, and physiology of human steroidogenesis and its disorders. Endocr Rev 32: 81-151, 2011.

3. Krone $\mathrm{N}$ and Arlt W: Genetics of congenital adrenal hyperplasia. Best Pract Res Clin Endocrinol Metab 23: 181-192, 2009.

4. Biason-Lauber A, Boscaro M, Mantero F and Balercia G: Defects of steroidogenesis. J Endocrinol Invest 33: 756-766, 2010.

5. Auchus RJ: The genetics, pathophysiology, and management of human deficiencies of P450c17. Endocrinol Metab Clin North Am 30: 101-119, vii, 2001.

6. Miller WL: Androgen synthesis in adrenarche. Rev Endocr Metab Disord 10: 3-17, 2009.
7. Miller WL: Steroid 17alpha-hydroxylase deficiency-not rare everywhere. J Clin Endocrinol Metab 89: 40-42, 2004.

8. Oh YK, Ryoo U, Kim D, Cho SY, Jin DK, Yoon BK, Lee DY and Choi D: 17alpha-hydroxlyase/17, 20-lyase deficiency in three siblings with primary amenorrhea and absence of secondary sexual development. J Pediatr Adolesc Gynecol 25: e103-e105, 2012.

9. Miller WL: Congenital adrenal hyperplasias. Endocrinol Metab Clin North Am 20: 721-749, 1991.

10. Kater CE and Biglieri EG: Disorders of steroid 17 alpha-hydroxylase deficiency. Endocrinol Metab Clin North Am 23: 341-357, 1994.

11. Yanase T, Simpson ER and Waterman MR: 17 alphahydroxylase/17,20-lyase deficiency: From clinical investigation to molecular definition. Endocr Rev 12: 91-108, 1991.

12. Biglieri EG, Herron MA and Brust N: 17-hydroxylation deficiency in man. J Clin Invest 45: 1946-1954, 1966.

13. Matteson KJ, Picado-Leonard J, Chung BC, Mohandas TK and Miller WL: Assignment of the gene for adrenal P450c17 (steroid 17 alpha-hydroxylase/17,20 lyase) to human chromosome 10 . J Clin Endocrinol Metab 63: 789-791, 1986.

14. Picado-Leonard J and Miller WL: Cloning and sequence of the human gene for P450c17 (steroid 17 alpha-hydroxylase/17,20 lyase): Similarity with the gene for P450c21. DNA 6: 439-448, 1987.

15. Miller WL: The syndrome of 17,20 lyase deficiency. J Clin Endocrinol Metab 97: 59-67, 2012.

16. Turcu AF and Auchus RJ: The next 150 years of congenital adrenal hyperplasia. J Steroid Biochem Mol Biol 153: 63-71, 2015.

17. Goldsmith O, Solomon DH and Horton R: Hypogonadism and mineralocorticoid excess. The 17-hydroxylase deficiency syndrome. N Engl J Med 277: 673-677, 1967.

18. Larson A, Nokoff NJ and Travers S: Disorders of sex development: Clinically relevant genes involved in gonadal differentiation. Discovery medicine 14: 301-309, 2012.

19. Philip J, Anjali, Thomas N, Rajaratnam S and Seshadri MS: 17-Alpha hydroxylase deficiency: An unusual cause of secondary amenorrhoea. Aust N Z J Obstet Gynaecol 44: 477-478, 2004.

20. Kim YM, Kang M, Choi JH, Lee BH, Kim GH, Ohn JH, Kim SY, Park MS and Yoo HW: A review of the literature on common CYP17A1 mutations in adults with 17-hydroxylase/17,20-lyase deficiency, a case series of such mutations among Koreans and functional characteristics of a novel mutation. Metabolism 63: 42-49, 2014.

21. Zhang M, Sun S, Liu Y, Zhang H, Jiao Y, Wang W and Li X: New, recurrent and prevalent mutations: Clinical and molecular characterization of 26 Chinese patients with 17alpha-hydroxylase/17,20-lyase deficiency. J Steroid Biochem Mol Biol 150: 11-16, 2015 .

22. Hahm JR, Kim DR, Jeong DK, Chung JH, Lee MS, Min YK, Kim KW and Lee MK: A novel compound heterozygous mutation in the CYP17 (P450 17alpha-hydroxylase) gene leading to 17alpha-hydroxylase/17,20-lyase deficiency. Metabolism 52: 488-492, 2003.

23. Fardella CE, Zhang LH, Mahachoklertwattana P, Lin D and Miller WL: Deletion of amino acids Asp487-Ser488-Phe489 in human cytochrome P450c17 causes severe 17 alpha-hydroxylase deficiency. J Clin Endocrinol Metab 77: 489-493, 1993.

24. Kim SM and Rhee JH: A case of 17 alpha-hydroxylase deficiency. Clin Exp Reprod Med 42: 72-76, 2015.

25. Marsh CA and Auchus RJ: Fertility in patients with genetic deficiencies of cytochrome P450c17 (CYP17A1): Combined 17-hydroxylase/17,20-lyase deficiency and isolated 17,20-lyase deficiency. Fertil Steril 101: 317-322, 2014.

26. Levran D, Ben-Shlomo I, Pariente C, Dor J, Mashiach S and Weissman A: Familial partial 17,20-desmolase and 17alpha-hydroxylase deficiency presenting as infertility. J Assist Reprod Genet 20: 21-28, 2003. 